\title{
Relevamiento de los contenidos curriculares de Ciencias de la Tierra en la formación de profesores de primaria de la ciudad de Buenos Aires
}

\author{
Diego Arias Regalía \\ Instituto de Investigación en Didáctica de las Ciencias \\ Naturales CEFIEC,Ciudad Universitaria (Pab.II) \\ Ciudad Autónoma de Buenos Aires - Argentina \\ dariasregalia@gmail.com \\ Leonor Bonan \\ Instituto de Investigación en Didáctica de las Ciencias \\ Naturales CEFIEC,Ciudad Universitaria (Pab.II) \\ Ciudad Autónoma de Buenos Aires - Argentina \\ Ibonan@de.fcen.uba.ar
}

\begin{abstract}
A SURVEY ON CONTENTS OF EARTH SCIENCE CURRICULUM FOR PRIMARY TEACHER TRAINING OF THE BUENOS AIRES CITY - This work focuses on the survey of the curriculum of the area of natural sciences in Buenos Aires, both in teacher training and elementary level. A brief analysis of the potential of this curriculum to be approached from a multidisciplinary perspective that promotes reflection on the nature of science, the purpose of scientific education, and the study of issues of social relevance is proposed. We found that the chosen selection of geological content is appropriate to develop the curriculum goals, allowing an inclusive approach. However, the lack of tradition in this view of education coupled with the lack of geological knowledge in middle school, impose large restrictions on what teachers can really do with these proposals. Citation: Arias Regalía D., Bonan L.. 2014. Relevamiento de los contenidos curriculares de ciencias de la Tierra en la formación de profesores de primaria de la Ciudad de Buenos Aires. Terræ Didatica, 10(3):455-460. http://www.ige.unicamp.br/terraedidatica/.
\end{abstract}

KEYWORDS: Teacher training, Elementary school, Earth Sciences teaching.

RESUMEN Este trabajo se centra en relevar los diseños curriculares del área de ciencias naturales en la Ciudad de Buenos Aires, tanto de la formación de maestros como del nivel primario para el que se forman. A su vez, se propone un breve análisis de la potencialidad de este recorte particular para ser abordado desde una perspectiva multidisciplinar que promueva la reflexión sobre la naturaleza de la ciencia, los fines de la educación científica, y el estudio de cuestiones de relevancia social. Encontramos que el recorte de contenidos geológicos elegido es apropiado para desarrollar los objetivos del diseño y para pensar una presentación de las ciencias naturales de carácter integrador. Sin embargo, la falta de tradición en esta perspectiva de la enseñanza sumada a la falta de circulación del conocimiento geológico en la escuela media, imponen restricciones muy grandes a la apropiación por parte de los docentes de estas propuestas.

PALABRAS CLAVE: Formación docente, Educación primaria, Enseñanza de las ciencias de la Tierra. 


\section{Introducción}

Las sucesivas reformas curriculares del sistema educativo argentino han ido desplazando los contenidos del área de las Ciencias de la Tierra fuera de los programas del nivel medio. Actualmente, su estudio se encara fundamentalmente en el nivel primario, cuyos docentes no tienen particular formación en el área, y la presencia de contenidos geológicos en el secundario se encuentra dentro de la asignatura Geografía, donde quedan diluidos en una materia que abarca una gran cantidad de temas, y que se entiende como una ciencia social. Como consecuencia de ello, no hay prácticamente circulación de conocimientos geológicos en la escuela media, con todo lo que esto conlleva desde el punto de vista del posicionamiento de la disciplina en el ideario de la sociedad, el nulo manejo de conceptos del área a la hora de analizar crítica e informadamente las cuestiones referentes a recursos naturales, sustentabilidad, riesgos geológicos, patrimonio geológico, impacto ambiental, etc., y la bajísima probabilidad de despertar vocaciones en los futuros científicos debido al desconocimiento de la existencia de la disciplina.

El problema de la falta de formación en geología (sus contenidos específicos, su práctica, su idiosincrasia y su didáctica) también alcanza a los propios Institutos de Formación Docente, ya que la mayoría de los profesores que dictan clases allí en este momento carecen a su vez de formación específica tanto en Ciencias de la Tierra como en su didáctica asociada.

En este marco, resulta útil disponer de un relevamiento de los contenidos de ciencias de la Tierra presentes en los diseños curriculares tanto de la formación de maestros como del nivel primario, como paso previo a la generación de propuestas de formación o actualización docente y de modos de intervención, que estén fuertemente sustentados en la investigación didáctica y sólidamente construidos sobre los ejes conceptualmente centrales del área (Bonan 2010).

Este trabajo se centra en relevar los diseños curriculares del área de ciencias naturales en la Ciudad de Buenos Aires, tanto de la formación de maestros como del nivel primario para el que se forman. A su vez, se propone un breve análisis de la potencialidad de este particular recorte para ser abordado desde una perspectiva multidisciplinar que vincule diversos saberes escolares y que, en el contexto de un programa más amplio de educación ambiental, promueva la reflexión sobre la naturaleza de la ciencia, los fines de la educación científica, la conexión entre la ciencia y la tecnología, y el estudio de aquellos conocimientos que tengan relevancia social (Bonan 2012, Sanmartí 2002).

\section{Organización del profesorado de primaria en el área de ciencias naturales}

La formación del profesorado de primaria en la Ciudad de Buenos Aires se desarrolla en instituciones terciarias no universitarias específicas, las Escuelas Normales Superiores (ENS).

Según el diseño curricular vigente (2009), tiene una duración de 3900 horas cátedra, distribuidas a lo largo de 4 años, y está organizada alrededor de 3 campos: Formación General, Formación Específica y Formación en las Prácticas Docentes.

El Campo de la Formación General se alimenta de distintas disciplinas (filosofía, historia, pedagogía, didáctica, política educativa, tecnología, psicología) y apunta a la construcción de marcos y perspectivas para analizar la actividad docente y las problemáticas de la enseñanza.

El Campo de la Formación Específica, formado por asignaturas como "Enseñanza de la matemática", "Alfabetización inicial", "Enseñanza de las ciencias sociales", que combinan aspectos disciplinares y didácticos, permite abordar la especificidad de la educación en el nivel a través del trabajo sobre la selección de contenidos, el uso del tiempo y de los espacios, la programación y el diseño de las actividades de enseñanza, la evaluación, etc.

El Campo de la Formación en las Prácticas Docentes apunta a la adquisición de instrumentos específicos vinculados al desempeño docente en los contextos reales de las instituciones educativas. Incluye talleres de práctica de la enseñanza, de diseño de actividades de enseñanza, y de residencia pedagógica en las escuelas asociadas.

La formación en el área de ciencias naturales se da a través de las materias cuatrimestrales Enseñanza de las Ciencias Naturales I, II y III del campo de la formación específica, que articulan contenidos disciplinares, epistemológicos y didácticos (En particular, los contenidos de ciencias de la Tierra aparecen fundamentalmente en la primera de ellas). Si bien desde lo conceptual el desarrollo de las 3 materias asume su escalonamiento, las Enseñanzas II y III no son correlativas entre sí, y solo lo son respecto de la Enseñanza I. 
Esta formación se continúa en el Taller de Diseño de Proyectos de Enseñanza de Ciencias Naturales del Campo de la Formación en las Prácticas Docentes, y en las asesorías curriculares de cada área que los estudiantes reciben durante los diversos períodos de práctica docente: los Talleres de Prácticas de la Enseñanza, y los Talleres de Residencia pedagógica. Estos talleres abordan progresivamente la intervención docente en los contextos reales de las escuelas asociadas.

\section{Las Ciencias de la Tierra en la formación de maestros}

Según los documentos curriculares vigentes (2004 para el nivel primario, 2009 para la formación de maestros), la enseñanza de las ciencias naturales debe articular conocimientos disciplinares con conocimientos epistemológico-didácticos, de modo de construir un conjunto de contenidos específicos que permitan concebir la ciencia desde una perspectiva humanista, integrada a nuestro acervo cultural, superando así la concepción cientificista que sostiene la neutralidad del conocimiento científico e invisibiliza los procesos y contextos sociohistóricos de su producción y aplicación, así como sus aspectos controversiales (ciencia y género, ciencia y valores, impacto ambiental, impacto sociocultural, etc.).

Los contenidos de ciencias de la Tierra aparecen fuertemente en la Enseñanza de las ciencias naturales I. Dentro de la perspectiva mencionada, esta primera asignatura de la serie de Enseñanza de las Ciencias Naturales tiene el doble propósito de presentar contenidos disciplinares relativos a la físico-química y a la geología, y a la vez desarrollar algunas herramientas básicas de la didáctica de las ciencias naturales (ideas previas, naturaleza de la ciencia, obstáculos didácticos y epistemológicos, etc.) que gozan de buen consenso actualmente dentro de la comunidad de didactas. Todo esto promoviendo el desarrollo de habilidades cognitivo-linguísticas que le permitan a los futuros docentes adaptarse a los desafíos que los constantes cambios en el mundo científico-tecnológico plantean respecto de qué y cómo enseñar.

La serie de materias Enseñanza de las ciencias naturales I, II y III se dictan de manera cuatrimestral, con una carga horaria de 6 horas cátedra semanales cada una, y están organizadas alrededor de tres ejes: uno de contenidos epistemológico-didácticos, otro de contenidos disciplinares, y otro de contenidos didácticos.
En el eje epistemológico-didáctico se propone fundamentalmente abordar las distintas concepciones de ciencia y sus implicancias en la enseñanza. Además, se trabaja sobre las relaciones entre las ideas previas y los obstáculos cognitivos o la gestación de las teorías implícitas de los estudiantes respecto de los fenómenos de incumbencia de las ciencias naturales.

En el eje de contenidos didácticos se pretende presentar y analizar el diseño curricular para la escuela primaria, a la luz de los enfoques actuales de la didáctica del área, haciendo hincapié en la discusión sobre la diferencia entre el conocimiento científico y el escolar, la validez y limitaciones del uso de modelos y analogías, y el sentido de la progresión de contenidos. Se propone también la reflexión sobre la relación entre los aspectos metodológicos de las prácticas de enseñanza y las concepciones de ciencia y de enseñanza que los subyacen.

Finalmente, los dos núcleos temáticos a desarrollar en el eje de contenidos disciplinares son la estructura de la materia y la estructura de la Tierra (y sus cambios en ambos casos), a los que se propone analizar desde la perspectiva de los modelos científicos actualmente aceptados para su comprensión: el modelo corpuscular de la materia y la tectónica de placas. En este caso, el foco está puesto en la relación de los contenidos con las teorías y los modelos explicativos, afrontando el salto cognitivo de pasar de una visión descriptiva a una explicativa de los fenómenos involucrados. Adicionalmente, parte del trabajo pasa por abordar la organización, secuenciación y alcances de los contenidos en cada año.

\section{Las Ciencias de la Tierra en la educación primaria}

El diseño curricular de la Ciudad de Buenos Aires para la educación primaria es de carácter prescriptivo, y organiza los contenidos para los siete años del ciclo alrededor de cuatro bloques: los materiales, los seres vivos, las fuerzas y el movimiento, y la Tierra y el Universo. Los contenidos geológicos aparecen a partir de $5^{\circ}$ grado (los últimos tres años de la educación primaria) dentro del bloque la Tierra y el Universo.

La tabla 1 está tomada del diseño curricular, e ilustra la progresión de conceptos a lo largo del 
ciclo (Posteriormente, para cada tema el diseño aborda la o las ideas básicas y el alcance de los contenidos a desarrollar).

Sin entrar en el análisis detallado de en qué año se ubica cada uno, y a partir de la lectura de las fundamentaciones, las recomendaciones metodológicas, los alcances y las ideas básicas que para los contenidos se desarrollan en el diseño, se puede elaborar la siguiente serie de temas, ideas y relaciones del área de las ciencias de la Tierra allí presentes

El planeta Tierra en el Sistema Solar:

- Modelos internos y externos: ¿̇cómo entender lo que se observa en el cielo?

- El "aspecto" de la Tierra visto desde el espacio.

- Las características comunes de los planetas que habilitan pensar en un origen común del Sistema Solar.

- Reconocimiento del calentamiento de la Tierra (la atmósfera, los océanos, el suelo) con relación a la inclinación con que llegan los rayos del Sol. Relación entre la inclinación del eje terrestre respecto del plano de la órbita y las estaciones.

- Dirección de la fuerza gravitatoria y su relación con las nociones de arriba y abajo.

- Magnitudes características y escalas:

- Importancia de las magnitudes características para comparar, estimar y clasificar.

La Tierra, su historia y sus cambios:

- El aspecto de la Tierra, desde su origen, continúa cambiando permanentemente como resultado de

Tabla 1: Bloque "La Tierra y el Universo"

\begin{tabular}{l|l|l}
\hline $5^{\circ}$ grado & $6^{\circ}$ grado & $7^{\circ}$ grado \\
\hline $\begin{array}{l}\text { La Tierra. } \\
\text {-Estructura y características de }\end{array}$ & $\begin{array}{l}\text { La Tierra. } \\
\text { la Tierra. }\end{array}$ & \\
-Longitudes características. & historia. & \\
& -Los restos fósiles. & \\
El cielo visto desde la Tierra. & & \\
-La Luna, satélite de la Tierra. & Magnitudes características. & \\
-Movimientos aparentes de las & & \\
estrellas. & & \\
El Sistema Solar. & & \\
-Descripción de los & El Universo. & El Universo. \\
componentes del Sistema & -Las galaxias. & -El Sistema Solar. \\
Solar. & artificiales. & -Las estaciones. \\
-Movimientos de los planetas. & & -Las fases de la Luna. \\
\hline
\end{tabular}

diversos procesos, que ocurren en muy variadas escalas temporales (Distinción entre procesos que modifican el paisaje lenta o violentamente, desde el punto de vista geológico).

- Eventos que, a lo largo de su historia, han ido modificando el aspecto de la Tierra. Glaciaciones, surgimiento de cadenas montañosas, separación de los continentes.

- Representaciones del tiempo geológico. Las eras geológicas y los principales cambios y eventos ocurridos.

- Interpretación de esquemas explicativos sobre la disposición de los estratos geológicos según el principio de superposición de estratos, sobre los cambios en la disposición de los continentes según el modelo de la tectónica de placas, y de la constitución y dinámica de la corteza terrestre.

\section{La potencialidad del temario de ciencias de la Tierra}

Diversos trabajos (Bonan 2006, Adúriz Bravo 2005, Frodeman 1995, Sequeiros 1994, Carrascosa et al. 1993, Pedrinaci 1992a, Gil Pérez 1991) sostienen que es indispensable incorporar en la formación docente, además del dominio de la disciplina, tanto cuestiones de Naturaleza e Historia de la Ciencia como conocimiento sobre los obstáculos conceptuales que presenta el aprendizaje de la disciplina misma.

A su vez, aparece reiteradamente el planteo de la necesidad de una enseñanza de las ciencias naturales que promueva la reflexión sobre la naturaleza de la ciencia, los fines de la educación científica, la conexión entre la ciencia y la tecnología o las implicaciones éticas y sociales de la ciencia, de modo de abordar el estudio de conocimientos que tengan relevancia social (Bonan 2012, Pedrinaci 2012, Gil Pérez \& Vilches 2006, Sanmartí 2002).

El desarrollo científico-tecnológico del mundo en el que vivi- 
mos tiene profundas implicancias en nuestra vida diaria: comunicaciones, transporte, producción de alimentos, medicina, explotación de recursos naturales, generación de energía, impacto ambiental, impacto sociocultural, distribución de la riqueza, acceso a los recursos, etc., y existe un cierto consenso dentro de la comunidad educativa respeto de la necesidad de lograr algún tipo de alfabetización científica, que tenga en cuenta que la ciencia es una actividad cultural de la humanidad, que se desarrolla en contextos históricos, sociales, culturales, políticos y económicos, que influyen directamente en su dinámica.

En esta parte del trabajo se pretende sugerir algunas pocas líneas de acción que tienen la potencialidad de servir como hilos conductores para resaltar que los contenidos de ciencias de la Tierra presentan relaciones que cruzan distintas unidades y áreas de la educación primaria, lo que permite pensar en abordajes que las pongan en diálogo.

Por ejemplo, poner la mirada sobre las escalas temporales y espaciales junto con el desarrollo de la idea de magnitudes características como "hilo conductor" de un abordaje interdisciplinario, ya que jugar con cambios de escala temporales y/o espaciales habilita la aparición de las distintas disciplinas escolares (o de distintos campos dentro de cada disciplina), y permite atravesar (pensando en la formación docente) gran variedad de situaciones con obstáculos cognitivos y/o concepciones alternativas conocidas.

Un posible punto de partida para trabajar desde la perspectiva mencionada es el de la formación del paisaje, que permite abordar diversos elementos importantes: pensar a partir de procesos; atacar la idea del paisaje estático; trabajar sobre escalas temporales y espaciales muy diversas; relacionar los cambios en el paisaje con su efecto sobre actividad biológica (dependiendo de la escala temporal trabajada, se puede ir desde evolución hasta la conformación de ecosistemas); estudiar la interacción entre el paisaje y la actividad humana; analizar procesos que afectan el paisaje en escalas de tiempo humanamente perceptibles y su relación con, por ejemplo, el riesgo geológico, la explotación de recursos naturales o el impacto ambiental; la relación entre los procesos formadores de pasaje y los grandes procesos que el marco teórico de la geología actual utiliza para entender la evolución del planeta (tectónica de placas, convección en el manto).
Incluir el abordaje histórico de la aceptación de la teoría de la deriva continental, el análisis de controversias como la del Actualismo vs Catastrofismo (P.ej. Pedrinaci 1992b), el desarrollo de la tectónica de placas, o la evolución del concepto de recurso natural desde su original sentido economicista centrado en la explotación hasta miradas que lo entienden como bien común de la naturaleza, permite poner de manifiesto cuestiones de naturaleza de la ciencia y de dinámica de la comunidad científica, o incorporar en la discusión cuestiones de relevancia social.

\section{Conclusiones}

La lectura de los documentos curriculares para la formación de docentes del nivel primario en ciencias naturales y, en particular, en ciencias de la Tierra, deja la sensación de encontrarse frente a una producción que guarda buena sintonía con las posiciones didácticas actuales. Además, como se ha intentado esbozar de manera breve en la sección anterior, el recorte de contenidos geológicos elegido es potencialmente potente para desarrollar los objetivos explícitos del diseño y para pensar una presentación de las ciencias naturales de carácter integrador.

Sin embargo, la realidad de la práctica docente indica que se está lejos de una materialización de los lineamientos básicos del diseño. La falta de tradición en una enseñanza que ponga el ojo en cuestiones de naturaleza de la ciencia, que discuta el propósito de la educación científica, que visibilice sus aspectos controversiales, o que pretenda ponerla al servicio del análisis crítico e informado de las problemáticas socialmente relevantes, sumada a la falta de circulación del conocimiento geológico en la escuela, imponen restricciones muy grandes a la apropiación por parte de los docentes de estas propuestas.

La formación de formadores, como dispositivo de intervención en el sistema educativo, necesita transformar las prácticas construyendo puentes entre lo que los profesores hacen y lo que se les propone (Perrenoud 2007), advirtiendo los límites de lo que pueden hacer en las condiciones que plantean los contextos escolares actuales y en virtud de los saberes de los que disponen (Terigi 2006).

Aparece como necesario entonces generar investigación didáctica que se traduzca en propuestas concretas de intervención con el fin de mejorar las prácticas de la enseñanza en ciencias naturales. Esta intervención debe implicar involu- 
crar a los docentes en la resolución holística de los problemas, convocando su interés, caracterizando los problemas de su entorno educativo y poniendo a su disposición las herramientas didácticas necesarias para realizar tal mejora (Bonan 2012). Hewson (2007) afirma que si los docentes no están involucrados, las reformas educativas no se producen.

\section{Bibliografía}

Adúriz Bravo A. 2005. Una introducción a la naturaleza de la ciencia. La epistemología en la enseñanza de las ciencias naturales. Buenos Aires: Fondo de Cultura Económica. 102p.

Bonan L. 2012. Desarrollo profesional en educación ambiental. Resignificación de estrategias de formación docente en ciencias naturales. Proyecto UBACyT 2012-2014 - Universidad de Buenos Aires. Inédito.

Bonan L. 2010. Desarrollo profesional en educación ambiental. Estrategias de formación en ciencias naturales. Proyecto UBACyT 2010-2012 - Universidad de Buenos Aires. Inédito.

Bonan L. 2006. La historia reciente de las Ciencias de la Tierra como estrategia de enseñanza para afrontar las representaciones epistemológicas ingenuas. Aveiro, Portugal: XIV Simposio sobre Enseñanza de la Geología. p125-130. (Ponencia oral).

Carrascosa J., Femández I., Gil D., Orozco A. 1993. Análisis de algunas visiones deformadas sobre la Naturaleza de la Ciencia y las características del trabajo científico. Rev. Enseñanza de las Ciencias, número extra (IV Congreso). p. 43-44.

Frodeman R. 1995. Geological reasoning: Geology as an interpretive and historical science. Bulletin
GSA, 107(8):960-968.

Gil Pérez D., Vilches A. 2006. Educación ciudadana y alfabetización científica: Mitos y Realidades. Revista Iberoamericana de Educación, 42:31-53.

Gil Pérez D. 1991. ¿Qué hemos de saber y saber hacer los profesores de Ciencias? Rev. Enseñanza de las Ciencias, 9(1):69-77.

Hewson P. 2007. Teacher Professional Development in Science. En: Abell S., Lederman N. eds. 2007. Handbook of Research on Science Education. New Jersey: LEA Publishers. p. 1.177-1.202.

Pedrinaci E. 2012. Alfabetización en Ciencias de la Tierra, una propuesta necesaria. Rev. Enseñanza de las Ciencias de la Tierra. 20(2):133-140.

Pedrinaci E. 1992a. Construcción histórica de los conceptos de cambio geológico, tiempo geológico y origen de las rocas. Aportaciones para su enseñanza y aprendizaje. Memoria Investigación curso doctorado, Univ. Sevilla. 160p. (inéd.).

Pedrinaci E. 1992b. Catastrofismo versus actualismo. Implicaciones didácticas. Rev. Enseñanza de las Ciencias de la Tierra. 10(2):216-222.

Perrenoud P. 2007. Desarrollar la práctica reflexiva en el oficio de enseñar. Profesionalización y razón pedagógica. Barcelona: Grao. 224p. Ed. Orig. en francés 2001. Paris: ESF éditeur.

Sanmartí N. 2002. Didáctica de las ciencias en la educación secundaria obligatoria. Madrid: Síntesis. 382p.

Sequeiros L. 1994. La formación del profesorado de geología: nuevos saberes y nuevas tareas. Rev. Enseñanza de las Ciencias de la Tierra. 2(2):318-325.

Terigi F. 2006. Tres problemas para las políticas docentes. En el Encuentro Internacional: La docencia, ¿una profesión en riesgo? Montevideo. URL: http://miguelricci2008.blogspot.com.ar/2010/09/ tres-problemas-para-las-politicas.html. Acceso 20.04.2013 